\title{
Possible performance improvement in [2]catenane molecular electronic switches
}

\author{
Yong-Hoon Kim ${ }^{\text {a) }}$ \\ Korea Institute for Advanced Study, 207-43 Cheongnyangni 2-dong, Dongdaemun-gu, \\ Seoul 130-722, Korea \\ Seung Soon Jang and William A. Goddard III \\ Materials and Process Simulation Center, California Institute of Technology, Pasadena, \\ California $91125-7400$
}

(Received 9 December 2005; accepted 2 March 2006; published online 20 April 2006)

\begin{abstract}
Mechanically interlocked bistable supramolecular complexes are promising candidates of molecular electronics. Applying a multiscale computational approach, here we study the coherent charge transport properties of catenane monolayers sandwiched between $\mathrm{Cu}(111)$ electrodes. We demonstrate the robust nature of electrical switching behavior with respect to the variations in the monolayer packing density and the type of electrodes, as well as the thermal fluctuations of the molecules. We propose that the asymmetry of molecule-electrode barriers can be utilized to improve the switching ratio. (C) 2006 American Institute of Physics. [DOI: 10.1063/1.2195087]
\end{abstract}

Generation of supramolecules, large molecular aggregates composed of subunits held together by noncovalent interactions, through molecular recognition and selfassembly represents a promising route toward nanotechnology. ${ }^{1}$ Prime examples are the [2]catenane and [2]rotaxane molecules ${ }^{2}$ in which an electron-accepting tetracationic cyclobis(paraquat- $p$-phenylene) $\left(\mathrm{CBPQT}^{4+}\right)$ cyclophane encloses either a ring-shaped or a linear backbone with two electron-donating stations, tetrathiafulvalene (TTF) and the 1,5-dioxynaphthalene (DNP) [Figs. 1(a) and 1(b)]. These molecules are designed to assume bistable conformations, $\mathrm{CBPQT}^{4+}$ encircling the stronger donor TTF as the ground state co-conformation (GSCC) and $\mathrm{CBPQT}^{4+}$ encircling the weaker donor DNP as the metastable state coconformation (MSCC) [Fig. 1(c)]. Solid-state tunnel junctions based on catenane and rotaxane Langmuir-Blodgett monolayers were constructed, and the reversible electrical switching in ambient conditions was demonstrated. While the voltage-gated conformational change of the molecules between the off-state GSCC and the on-state MSCC was proposed as the switching mechanism, ${ }^{3-6}$ the molecular origin of the switching was questioned ${ }^{7}$ as seemingly contradictory experiments appeared. ${ }^{8-10}$ Indeed, differentiating a molecularly inherent switching from a stochastic one is a difficult task, ${ }^{11}$ because the charge transport in molecular scale junctions is strongly influenced by the moleculeelectrode contacts and conformational fluctuations of molecules. ${ }^{12-15}$

In a recent work, ${ }^{16}$ employing realistic catenane monolayer models sandwiched between $\mathrm{Au}(111)$ electrodes, we explicitly showed that the electrical switching (between on and off) can originate from the energetic movement of frontier orbitals that accompanies the structural switching of the molecule (between GSCC and MSCC, respectively). However, there exist many potential noises in the device, so the molecularly inherent nature of the switching signal still needs to be demonstrated. In this letter, we assess the reliability of the identified electrical switching mechanism using

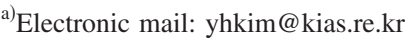

a catenane device model that differs from the previous one in terms of the packing density and the type of electrodes as well as taking into account thermal fluctuations of the molecules. We, in particular, consider the role of moleculeelectrode barriers that should play an important role in the experimental situation, ${ }^{8}$ and propose that the switching ratio of the device can be significantly improved by increasing the asymmetry of the interface barriers.

In our study, as in the experiments, ${ }^{2,17}$ we first obtained a molecular configuration at an optimal monolayer packing density. ${ }^{16,18}$ For this purpose, we used the CERIUS2 program (Accelrys) to perform classical molecular dynamics (MD) simulations based on the extended generic Dreiding force field ${ }^{18,19}$ and charges from the charge equilibration model. ${ }^{20}$ The accuracy of these force fields and details of the modeling approach have been described previously. ${ }^{18}$ Shortly, we constructed models at varying packing densities by using $m \times n \quad(m, n=3-7) \quad \mathrm{Au}(111) \quad$ supercell slabs as the template. ${ }^{16,18}$ Fixing the Au electrode atoms, we first conducted canonical ensemble (NVT) MD simulations at $300 \mathrm{~K}$ for $100 \mathrm{ps}$ with $1 \mathrm{fs}$ time step to preequilibrate the system. Then, we performed another 200 ps NVT MD simulations to extract the molecular structure for the device modeling. We identified the $4 \times 4-4 \times 5$ Au (111) grid/molecule (115-144 $\AA^{2} /$ molecule) as the optimal monolayer packing density ${ }^{16}$ in good agreement with the experimental value of $120 \AA^{2} /$ molecule. ${ }^{2,17}$ In modeling the monolayer, we replaced dimyristoylphosphatidyl $\left(\mathrm{DMPA}^{-}\right)$anions by $\mathrm{PF}_{6}^{-}$ anions. ${ }^{21}$ We found that dimensional changes between GSCC and MSCC are minimal, indicating that the structural transformation between the two conformations would be possible within the solid-state device setting, even when the molecules are confined between electrodes.

Next, to compare with our previous model based on the $4 \times 4-\mathrm{Au}(111)$ electrodes $\left(115 \AA^{2}\right)$ with the electrodeelectrode gap distance of $22 \AA$, we prepared a device model based on the three-layer $5 \times 5-\mathrm{Cu}(111)$ slabs $\left(141 \AA^{2}\right)$ with the gap distance of $21 \AA$. GSCC and MSCC monolayers on the $4 \times 4-\mathrm{Au}(111)$ slab were adopted as the initial configurations, and additional force-field MD simulations were per- 
(a)

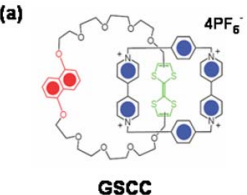

(b)

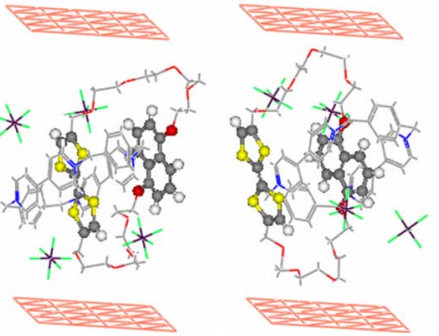

FIG. 1. (Color online) (a) Molecular drawing of the GSCC and MSCC catenanes. (b) Representative geometry of the $5 \times 5-\mathrm{Cu}(111)$-cell GSCC and MSCC catenane device models.

formed to equilibrate the systems. For the completed device models, we carried out pseudopotential ${ }^{22}$ Gaussian basis set density-functional calculations within the generalized gradient approximation ${ }^{23}$ using the SEQQUEST program (Sandia National Laboratories). A complex single reciprocal-space $\mathbf{k}$ point was sampled along the electrode-surface direction.

Finally, we carried out matrix Green's function calculations to compute the transmission function $T .^{15,16,24}$ Note that we included a large number of $\mathrm{Cu}$ electrode atoms (150 total) in our models to accurately estimate the energetic location of the transmission peaks with respect to the equilibrium Fermi level $E_{F}$. The broadening and shift of transmission peaks due to the coupling of molecules to the electrodes are also accurately described via self-energies. ${ }^{24}$ Four $\mathbf{k}$ points were sampled along the electrode-normal direction in the densityfunctional calculations of electrodes to construct the surface Green's functions that generate the self-energies. In calculating $T$, the energy was first scanned around $E_{F}$ using $0.01 \mathrm{eV}$ steps, and then the resolution was adaptively increased near the transmission resonances up to $0.0001 \mathrm{eV} .{ }^{16}$ With $T$, the current-voltage $(I-V)$ characteristics were obtained via the Landauer-type formula,

$$
I(V)=\frac{2 e}{h} \int_{\mu_{1}}^{\mu_{2}} d E T(E, V)\left[f\left(E-\mu_{1}\right)-f\left(E-\mu_{2}\right)\right],
$$

where $\mu_{1}$ and $\mu_{2}$ are the chemical potentials of the electrodes 1 and 2 , and $f$ is the Fermi-Dirac distribution function. ${ }^{15,16}$ In the experiments of Refs. 3-5, molecules were insulated from the poly-Si bottom electrode by a $\mathrm{SiO}_{2}$ layer and from the $\mathrm{Ti} / \mathrm{Al}$ top electrode by $\mathrm{DMPA}^{-}$anions (and probably a layer of titanium carbide), which implies that the electrostatic potential drop over the molecular core is negligible when the device is probed by nonperturbative small voltages. Since we did not include these insulating layers in our calculations, we instead assumed $T(E, V) \approx T(E, 0)$ and considered the role of the molecule-electrode barriers by setting $\mu_{1}=E_{F}-\eta e V$ and $\mu_{2}=E_{F}+(1-\eta) e V$ and varying $\eta$ from 0 (completely asymmetric barriers) to 0.5 (symmetric barriers). ${ }^{25}$

We first characterize the device properties for the representative GSCC and MSCC configurations.

(1) The charge conduction near $E_{F}$ is dominated by four resonant transmission channels [Fig. 2(a)]. Especially, the transmission peaks corresponding to the two highest occupied molecular orbitals (HOMO and HOMO-1)
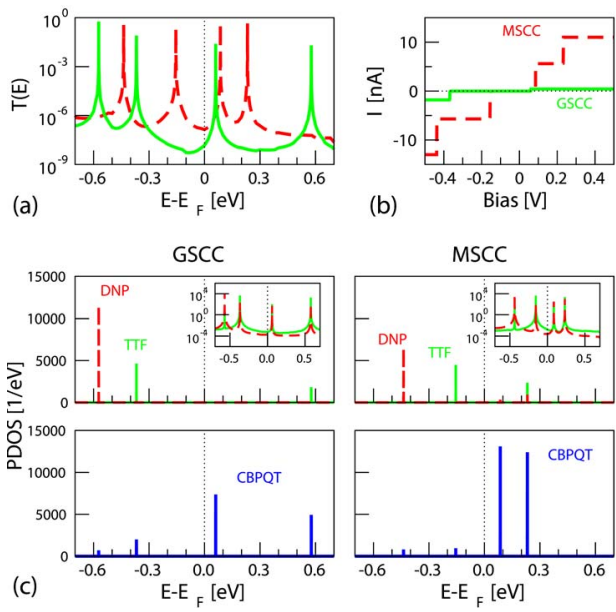

FIG. 2. (Color online) (a) Transmission and (b) $I-V$ characteristics of GSCC (solid lines) and MSCC (dashed lines) devices. (c) Upper panels: PDOS of TTF (solid lines) and DNP (dashed lines). Also shown in the logarithmic scale in the insets. Lower panels: PDOS of $\mathrm{CBPQT}^{4+}$.

shift upward and closer to $E_{F}$ (by $0.14 \mathrm{eV}$ for this case) and the second lowest unoccupied orbital (LUMO+1) shift downward as the relative position of $\mathrm{CBPQT}^{4+}$ changes from the TTF site (GSCC) to the DNP site (MSCC).

(2) The smaller HOMO-LUMO gap and larger transmission peaks in MSCC result in a larger current in MSCC (on) than in GSCC (off) when the device is probed by a small bias [Fig. 2(b)].

(3) The four transmission peaks correspond to the frontier orbitals of the redox-active components of the molecule: the HOMO from TIF, the HOMO-1 from DNP, and the LUMO and LUMO+1 from CBPQT ${ }^{4+}$ [Fig. 2(c)].

In Ref. 16, we have identified the origin of the systematic energetic movement of frontier orbital levels as follows: (i) $\mathrm{CBPQT}^{4+}$, being an electron acceptor, exerts a positive electrostatic potential on the encircled electron donor component and downshifts its frontier orbital levels. When they are interrogated by a small bias, this translates into the reduction of the current flow through the encircled backbone compared to the bare backbone. (ii) Because TTF is a stronger electron donor than DNP, TTF projected density-of-states (PDOS) lies higher and closer to $E_{F}$ than DNP PDOS. (iii) Also, upon encircling TTF and DNP by $\mathrm{CBPQT}^{4+}$, the $\mathrm{CBPQT}^{4+}-\mathrm{TTF}$ coupling is stronger than that of the $\mathrm{CBPQT}^{4+}$-DNP counterpart. This results in the bigger downshift of two HOMO levels and bigger splitting between two LUMO levels in MSCC than in GSCC, as described in the item (1). Note that the universality of our analysis was yet to be shown, so here we have proved its generality with respect to the changes in the monolayer packing density and electrode materials.

To further investigate the robustness of the identified switching mechanism and its relevance for the experiments performed in ambient conditions, we next considered the influence of thermal fluctuations of molecules on the device characteristics. Such statistical consideration is essential in molecular electronics where the flexible nature of molecules and the indeterminacy of molecule-electrode interfaces significantly hamper the identification of a truly molecular switching signal. ${ }^{11}$ For each GSCC and MSCC configuration, to AIP license or copyright, see http://apl.aip.org/apl/copyright.jsp 

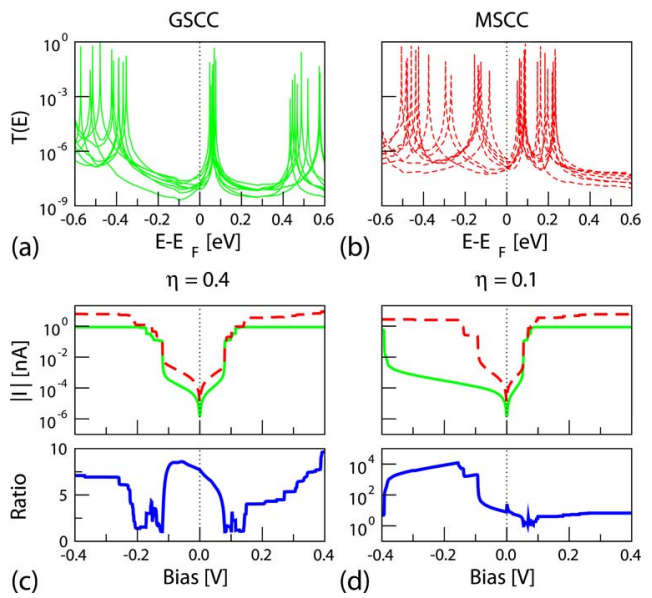

FIG. 3. (Color online) Transmission composites of eight (a) GSCC and (b) MSCC MD snapshots. Average $I-V$ characteristics of the GSCC (solid lines) and MSCC (dashed lines) devices, and the resulting switching ratios at (a) $\eta=0.4$ and (b) $\eta=0.1$.

we carried out two independent MD simulations and from each run we selected four random configurations at the $10 \mathrm{ps}$ time interval to prepare eight molecular structures. As shown in Figs. 3(a) and 3(b), the height and position of the resonance peaks fluctuate from configuration to configuration throughout the MD runs. The average $I-V$ data and switching ratios are presented in Figs. 3(c) and 3(d) at two different $\eta$ values, 0.1 and 0.4 . While the transmission peaks in each configuration fluctuate by $\sim 0.2-0.3 \mathrm{eV}$, we still find a robust on/off switching behavior. This results from the strongly resonant nature of the transmission peaks which should in turn results from the strong identity of the molecules and the rather weak coupling of their frontier orbitals with electrodes. This might represent a unique and general advantage of employing supramolecules for molecular electronics.

Before discussing Fig. 3 further, we emphasize that a mandatory precondition of observing an electronic switching at a small "read" bias $(-0.2-+0.2 \mathrm{~V})$, the focus of the current letter, is the mechanical switching of the molecules between GSCC and MSCC induced by applying a large "write" bias $(\sim \pm 2 \mathrm{~V})$. It was demonstrated that the electromechanical switching occurs within a variety of physical environments. ${ }^{3-6,26}$ However, it was also observed that an intrinsically molecular switching cannot be observed in solid-state experiments based on purely metallic electrodes, ${ }^{8,9}$ which signifies that the molecule-electrode barriers are required in the tunnel junction experiments. One possible reason is the formation of metallic filaments that would mask any molecular signature when the molecules are directly contacted to the electrodes. ${ }^{10}$ We now argue that the existence of molecule-electrode barriers is also important for the device function at a small bias. Remind that our calculations assumed the existence of barriers and the barrier asymmetry was simulated via $\eta$ [Eq. (1)]. While we obtain a robust yet nominal switching ratio of about 8 (in good agreement with experiments) at a small barrier asymmetry $\eta=0.4$ [Fig. 3(a)], we find at a large barrier asymmetry $\eta=0.1$ a significant enhancement of the switching ratio to the order of $10^{4}$ in the bias range from about -0.4 to $-0.1 \mathrm{~V}$ [Fig. 3(b)]. This is because in the catenane device the HOMO levels shift up and down following the mechanical switching of the molecules, while the LUMO is approximately fixed with respect to $E_{F}$. Note that this identification Downloaded 06 May 2006 to 131.215.240.9. Redistribution subject is based on the absolute location of frontier orbitals with respect to $E_{F}$ rather than the relative size of the HOMOLUMO gap. This demonstrates the importance of modeling molecules and electrodes as a single entity for the quantitative prediction of device properties.

In summary, we demonstrated for the catenane device a robust nature of the electrical switching behavior and pointed out a possibility of dramatically increasing the switching ratio by exploiting the molecule-electrode barriers.

One of the authors (Y.-H.K.) gratefully acknowledge Amar Flood for insightful discussions. He was supported by KRF-2005-041-C00125. Two of the authors (W.A.G. III and S.S.J.) were supported by NSF-NIRT, MARCO-FENA, ONR-DURIP, ARO-DURIP, and NSF-MRI.

${ }^{1}$ J.-M. Lehn, Proc. Natl. Acad. Sci. U.S.A. 99, 4763 (2002).

${ }^{2}$ P. M. Mendes, A. H. Flood, and J. F. Stoddart, Appl. Phys. A 80, 1197 (2005).

${ }^{3}$ C. P. Collier, G. Mattersteig, E. W. Wong, Y. Luo, K. Beverly, J. Sampaio, F. M. Raymo, J. F. Stoddart, and J. R. Heath, Science 289, 1172 (2000).

${ }^{4}$ C. P. Collier, J. O. Jeppesen, Y. Luo, J. Perkins, E. W. Wong, J. R. Heath, and J. F. Stoddart, J. Am. Chem. Soc. 123, 12632 (2001).

${ }^{5}$ Y. Luo, C. P. Collier, J. O. Jeppesen, K. A. Nielsen, E. DeIonno, G. Ho, J. Perkins, H.-R. Tseng, T. Yamamoto, J. F. Stoddart, and J. R. Heath, ChemPhysChem 3, 519 (2002).

${ }^{6}$ M. R. Diehl, D. W. Steuerman, H.-R. Tseng, S. A. Vignon, A. Star, P. C. Celestre, J. F. Stoddart, and J. R. Heath, ChemPhysChem 4, 1335 (2003).

${ }^{7}$ R. F. Service, Science 302, 556 (2003); J. R. Heath, J. F. Stoddart, and R. S. Williams, ibid. 303, 1136 (2004).

${ }^{8}$ H. Yu, K. Beverly, J. F. Stoddart, H.-R. Tseng, and J. R. Heath, Angew. Chem., Int. Ed. 42, 5706 (2003).

${ }^{9}$ D. R. Stewart, D. A. A. Ohlberg, P. A. Beck, Y. Chen, R. S. Williams, J. O. Jeppesen, K. A. Nielsen, and J. F. Stoddart, Nano Lett. 4, 133 (2004).

${ }^{10}$ C. N. Lau, D. R. Stewart, R. S. Williams, and M. Bockrath, Nano Lett. 4, 569 (2004).

${ }^{11}$ A. S. Blum, J. G. Kushmerick, D. P. Long, C. H. Patterson, J. C. Yang, J. C. Henderson, Y. Yao, J. M. Tour, R. Shashidhar, and B. R. Ratna, Nat. Mater. 4, 167 (2005).

${ }^{12}$ Z. J. Donhauser, B. A. Mantooth, K. F. Kelly, L. A. Bumm, J. D. Monnell, J. J. Stapleton, D. W. Price, Jr., A. M. Rawlett, D. L. Allara, J. M. Tour, and P. S. Weiss, Science 292, 2303 (2001).

${ }^{13}$ J. Taylor, M. Brandbyge, and K. Stokbro, Phys. Rev. Lett. 89, 138301 (2002).

${ }^{14}$ X. Xiao, B. Xu, and N. J. Tao, Nano Lett. 4, 267 (2004).

${ }^{15}$ Y.-H. Kim, S. S. Jang, and W. A. Goddard III, J. Chem. Phys. 123, 244703 (2005).

${ }^{16}$ Y.-H. Kim, S. S. Jang, Y. H. Jang, and W. A. Goddard III, Phys. Rev. Lett. 94, 156801 (2005).

${ }^{17}$ M. Asakawa, M. Higuchi, G. Mattersteig, T. Nakamura, A. R. Pease, F. M. Raymo, T. Shimizu, and J. F. Stoddart, Adv. Mater. (Weinheim, Ger.) 12, 1099 (2000).

${ }^{18}$ S. S. Jang, Y. H. Jang, Y.-H. Kim, W. A. Goddard III, A. H. Flood, B. W. Laursen, H.-R. Tseng, J. F. Stoddart, J. O. Jeppesen, J. W. Choi, D. W. Steurman, E. Delonno, and J. R. Heath, J. Am. Chem. Soc. 127, 1563 (2005); S. S. Jang, Y. H. Jang, Y.-H. Kim, W. A. Goddard III, J. W. Choi, J. R. Heath, B. W. Laursen, A. H. Flood, and J. F. Stoddart, ibid. 127, 14804 (2005).

${ }^{19}$ S. L. Mayo, B. D. Olafson, and W. A. Goddard III, J. Phys. Chem. 94, 8897 (1990)

${ }^{20}$ A. K. Rappé and W. A. Goddard III, J. Phys. Chem. 95, 3358 (1991).

${ }^{21}$ Since the role of $\mathrm{DMPA}^{-}$aions is to maintain the flat position of the $\mathrm{CBPQT}^{4+}$ plane of the molecules (Ref. 17), in replacing $\mathrm{DMPA}^{-}$by $\mathrm{PF}_{6}^{-}$, we applied the consraint on the unit cell such that the upright position of the molecules is maintained.

${ }^{22}$ M. Fuchs and M. Scheffler, Comput. Phys. Commun. 119, 67 (1999).

${ }^{23}$ J. P. Perdew, K. Burke, and M. Ernzerhof, Phys. Rev. Lett. 77, 3865 (1996).

${ }^{24}$ S. Datta, Electronic Transport in Mesoscopic Systems (Cambridge University Press, Cambridge, 1995).

${ }^{25}$ S. Datta, W. Tian, S. Hong, R. Reifenberger, J. I. Henderson, and C. P. Kubiak, Phys. Rev. Lett. 79, 2530 (1997).

${ }^{26}$ A. H. Flood, A. J. Peters, S. A. Vignon, D. W. Steuerman, H.-R. Tseng, S. Kang, J. R. Heath, and J. F. Stoddart, Chem.-Eur. J. 10, 6558 (2004). 